\title{
On the Differential Geometry of $G L_{q}(1 \mid 1)$
}

\author{
Salih Celik* ${ }^{*}$ and Sultan A. Celik ${ }^{+}$? \\ * Mimar Sinan University, Department of Mathematics, 80690 Besiktas, Istanbul, TURKEY. \\ + Yildiz Technical University, Department of Mathematics, 80270 Sisli, Istanbul, TURKEY.
}

\begin{abstract}
The differential calculus on the quantum supergroup $\mathrm{GL}_{q}(1 \mid 1)$ was introduced by Schmidke et al. (1990 Z. Phys. C 48 249). We construct a differential calculus on the quantum supergroup $\mathrm{GL}_{q}(1 \mid 1)$ in a different way and we obtain its quantum superalgebra. The main structures are derived without an R-matrix. It is seen that the found results can be written with help of a matrix $\hat{R}$
\end{abstract}

\footnotetext{
${ }^{1}$ E-mail: scelik@fened.msu.edu.tr

${ }^{2}$ E-mail: celik@yildiz.edu.tr
} 


\section{Introduction}

During the past few years, the theory of quantum groups [1] has been an important branch of mathematical physics and a new branch of mathematics. A quantum group is not a group in ordinary sense of this word. But a quantum group is somehow related to a group in the sense that it is a deformation of a certain structure reflecting the group properties. In other words, a quantum group coincides with the group for particular values of the deformation parameter. Quantum (super) groups present the examples of (graded) Hopf algebras. They have found application in as diverse areas of physics and mathematics as conformal field theory, statistical mechanics, nonlinear integrable models, knot theory and solutions of Yang-Baxter equations [2] (and references therein). Many of the remarkable properties of Matrix Theory appear to be closely connected to the ideals of noncommutative geometry [3]. More recently it has been suggested that the zero branes in M-theory should be identified with supercoordinates in noncommutative geometry [4].

Quantum (super) groups can be realized on a quantum (super) space in which coordinates are noncommuting [5]. Recently the differential calculus on noncommutative space has been intensively studied both by mathematicians and mathematical physicists. There is much activity in differential geometry on quantum groups. A noncommutative differential calculus on the quantum groups has been developed by Woronowicz [6] along the lines of the general ideas of Connes [3]. Wess and Zumino [7] have reformulated the general theory in an abstract way. A few other methods to construct a noncommutative differential geometry on a quantum group have been proposed and discussed by several authors (e.g. Refs 8).

In Ref. 9 a right-invariant differential calculus on the quantum supergroup $G L_{q}(1 \mid 1)$ has been constructed and it has been shown that the quantum Lie algebra generators satisfy the undeformed Lie superalgebra. In this paper we present a differential calculus on the quantum supergroup $G L_{q}(1 \mid 1)$ in a different way. This differential structure turns out to be a differential (graded) Hopf algebra. Although all of the commutation relations among the one-forms of Ref. 9 are classical, i.e. 'undeformed' in the present work they are $q$ deformed. It is also cited that the obtained relations can be written with help of a matrix $\hat{R}$.

In this work, we shall use greek letters to denote fermionic (odd) and latin letters to denote bosonic (even) variables. 


\section{Formalities}

Elementary properties of the quantum supergroup $G L_{q}(1 \mid 1)$ are described in $[10,11]$. We state briefly the properties we are going to need in this work.

The quantum supergroup $G L_{q}(1 \mid 1)$ is defined by, as a group element, the matrices of the form

$$
T=\left(\begin{array}{ll}
a & \beta \\
\gamma & d
\end{array}\right)
$$

where the matrix elements satisfy the following commutation relations $[10,11]$

$$
\begin{array}{cc}
a \beta=q \beta a & d \beta=q \beta d \\
a \gamma=q \gamma a & d \gamma=q \gamma d \\
\beta \gamma+\gamma \beta=0 & \beta^{2}=0=\gamma^{2} \\
a d=d a+\left(q-q^{-1}\right) \gamma \beta .
\end{array}
$$

Let us denote the algebra generated by the elements $a, \beta, \gamma, d$ with the relations $(2)$ by $\mathcal{A}$. We know that the algebra $\mathcal{A}$ is a (graded) Hopf algebra with the following structure:

(1) The usual coproduct

$$
\Delta: \mathcal{A} \longrightarrow \mathcal{A} \otimes \mathcal{A} \quad \Delta(T)=T \dot{\otimes} T
$$

(2) the counit

$$
\varepsilon: \mathcal{A} \longrightarrow \mathcal{C} \quad \varepsilon(T)=I
$$

(3) the coinverse (antipode)

$$
S: \mathcal{A} \longrightarrow \mathcal{A} \quad S(T)=\left(\begin{array}{cc}
a^{-1}+a^{-1} \beta d^{-1} \gamma a^{-1} & -a^{-1} \beta d^{-1} \\
-d^{-1} \gamma a^{-1} & d^{-1}+d^{-1} \gamma a^{-1} \beta d^{-1}
\end{array}\right)
$$

It is not difficult to verify the following properties of the co-structures:

$$
\begin{gathered}
(\Delta \otimes \mathrm{id}) \circ \Delta=(\mathrm{id} \otimes \Delta) \circ \Delta \\
\mu \circ(\varepsilon \otimes \mathrm{id}) \circ \Delta=\mu^{\prime} \circ(\mathrm{id} \otimes \varepsilon) \circ \Delta \\
m \circ(S \otimes \mathrm{id}) \circ \Delta=\varepsilon=m \circ(\mathrm{id} \otimes S) \circ \Delta
\end{gathered}
$$

where id denotes the identity mapping,

$$
\mu: \mathcal{C} \otimes \mathcal{A} \longrightarrow \mathcal{A} \quad \mu^{\prime}: \mathcal{A} \otimes \mathcal{C} \longrightarrow \mathcal{A}
$$


are the canonical isomorphisms, defined by

$$
\mu(k \otimes a)=k a=\mu^{\prime}(a \otimes k) \quad \forall a \in \mathcal{A} \quad \forall k \in \mathcal{C}
$$

and $m$ is the multiplication map

$$
m: \mathcal{A} \otimes \mathcal{A} \longrightarrow \mathcal{A} \quad m(a \otimes b)=a b
$$

The multiplication in $\mathcal{A} \otimes \mathcal{A}$ follows the rule

$$
(A \otimes B)(C \otimes D)=(-1)^{p(B) p(C)} A C \otimes B D
$$

where $p(X)$ is the $z_{2}$-grade of $X$, i.e. $p(X)=0$ for even variables and $p(X)=1$ for odd variables.

In the following section we shall build up the differential calculus on the quantum supergroup $G L_{q}(1 \mid 1)$. We first note that the properties of the exterior differential. The exterior differential $d$ is an operator which gives the mapping from the generators of $\mathcal{A}$ to the differentials:

$$
\mathrm{d}: u \longrightarrow \mathrm{d} u \quad u \in\{a, \beta, \gamma, d\}
$$

We demand that the exterior differential has to satisfy two properties: the nilpotency

$$
\mathrm{d}^{2}=0
$$

and the graded Leibniz rule

$$
\mathrm{d}(f g)=(\mathrm{d} f) g+(-1)^{p(f)} f(\mathrm{~d} g)
$$

We now introduce the algebra $\hat{\mathcal{A}}$ generated by the matrix elements $\alpha, b$, $c, \delta$ of $\hat{T}$,

$$
\hat{T}=\left(\begin{array}{ll}
\alpha & b \\
c & \delta
\end{array}\right)
$$

where the matrix elements $\alpha, b, c, \delta$ satisfy the commutation relations [12]

$$
\begin{gathered}
\alpha b=q^{-1} b \alpha \quad \alpha c=q^{-1} c \alpha \\
\delta b=q^{-1} b \delta \quad \delta c=q^{-1} c \delta \\
\alpha \delta+\delta \alpha=0 \quad \alpha^{2}=0=\delta^{2} \\
b c=c b+\left(q-q^{-1}\right) \delta \alpha .
\end{gathered}
$$

We shall use these relations in the following section. 


\section{Differential Geometric Structure of $G L_{q}(1 \mid 1)$}

We have seen, in the previous section, that $\mathcal{A}$ is an associative algebra (essentially a graded Hopf algebra) generated by the matrix elements of (1) with the relations (2). A differential algebra on $\mathcal{A}$ is a $z_{2}$-graded associative algebra $\Omega$ equipped with a linear operator $d$ given (11)-(13). Furthermore the algebra $\Omega$ has to be generated by $\Omega^{0} \cup \mathrm{d} \Omega^{0}$, where $\Omega^{0}$ is isomorphic to $\mathcal{A}$.

Firstly, we observe that the matrix elements of $\hat{T}$ are given by (14) just as the differentials of the matrix elements of $T$ are given by (1). Thus, as in the considerations for the quantum planes in [7], we can identify, at least formally,

$$
\hat{T}=\mathrm{d} T \text {. }
$$

So, let us rewrite the relations (15) in the form

$$
\begin{array}{rc}
\mathrm{d} a \mathrm{~d} \beta=q^{-1} \mathrm{~d} \beta \mathrm{d} a & \mathrm{~d} d \mathrm{~d} \beta=q^{-1} \mathrm{~d} \beta \mathrm{d} d \\
\mathrm{~d} a \mathrm{~d} \gamma=q^{-1} \mathrm{~d} \gamma \mathrm{d} a & \mathrm{~d} d \mathrm{~d} \gamma=q^{-1} \mathrm{~d} \gamma \mathrm{d} d \\
\mathrm{~d} a \mathrm{~d} d=-\mathrm{d} d \mathrm{~d} a & (\mathrm{~d} a)^{2}=0=(\mathrm{d} d)^{2} \\
\mathrm{~d} \beta \mathrm{d} \gamma=\mathrm{d} \gamma \mathrm{d} \beta+\left(q-q^{-1}\right) \mathrm{d} d \mathrm{~d} a .
\end{array}
$$

We now denote by $\mathcal{A}_{a \beta}$ the algebra generated by the elements $a$ and $\beta$ with the relations

$$
a \beta=q \beta a \quad \beta^{2}=0 .
$$

A possible set of commutation relations between the generators of $\mathcal{A}_{a \beta} \simeq \Omega_{a \beta}^{0}$ and $\hat{\mathcal{A}}_{\mathrm{d} a \mathrm{~d} \beta} \simeq \mathrm{d} \Omega_{\mathrm{d} a \mathrm{~d} \beta}^{0}$ has of the form

$$
\begin{gathered}
a \mathrm{~d} a=A \mathrm{~d} a a \\
a \mathrm{~d} \beta=F_{11} \mathrm{~d} \beta a+F_{12} \mathrm{~d} a \beta \\
\beta \mathrm{d} a=F_{21} \mathrm{~d} a \beta+F_{22} \mathrm{~d} \beta a \\
\beta \mathrm{d} \beta=B \mathrm{~d} \beta \beta .
\end{gathered}
$$

The coefficients $A, B$ and $F_{i j}$ will be determined in term of complex deformation parameter $q$. To find them we shall use the consistency of calculus. From the consistency conditions

$$
\mathrm{d}(a \beta-q \beta a)=0 \quad \mathrm{~d}\left(\beta^{2}\right)=0
$$

and

$$
(a \beta-q \beta a) \mathrm{d} a=0 \quad(a \beta-q \beta a) \mathrm{d} \beta=0
$$


we find

$$
F_{11}+q F_{22}=q \quad F_{12}+q F_{21}=-1 \quad B=1
$$

and

$$
F_{12} F_{22}=0=\left(F_{11}-q A\right) F_{22} .
$$

In fact (22) and (23) are, in disguise, the linear and quadratic consistency conditions similar to the ones discussed in full generality for quantum planes in [7]. Equation (23) admits two solutions. If we choose $F_{22}=0$ we are led to the following commutation relations

$$
\begin{gathered}
a \mathrm{~d} a=q^{2} \mathrm{~d} a a \\
a \mathrm{~d} \beta=q \mathrm{~d} \beta a+\left(q^{2}-1\right) \mathrm{d} a \beta \\
\beta \mathrm{d} a=-q \mathrm{~d} a \beta \\
\beta \mathrm{d} \beta=\mathrm{d} \beta \beta
\end{gathered}
$$

where $A$ equal to $q^{2}$ since this leads to the standard R-matrix (eq. (51)).

We denote by $\Omega_{a \beta}=\mathcal{A}_{a \beta} \cup \mathrm{d} \mathcal{A}_{\mathrm{d} a \mathrm{~d} \beta}$, the algebra generated by the generators $a, \beta$ (of $\mathcal{A}$ ) and the generators $\mathrm{d} a, \mathrm{~d} \beta$ (of $\mathrm{d} \mathcal{A}$ ) with the relations (18), a parity of (17) and (24a).

Continuing in this way, we can construct the differential algebra $\Omega_{a \gamma}, \Omega_{\beta \gamma}$, etc. The final result is given by

$$
\begin{gathered}
a \mathrm{~d} \gamma=q \mathrm{~d} \gamma a+\left(q^{2}-1\right) \mathrm{d} a \gamma \\
a \mathrm{~d} d=\mathrm{d} d a+\left(q-q^{-1}\right)\left[\mathrm{d} \gamma \beta-\mathrm{d} \beta \gamma+\left(q-q^{-1}\right) \mathrm{d} a d\right] \\
\beta \mathrm{d} \gamma=\mathrm{d} \gamma \beta+\left(q-q^{-1}\right) \mathrm{d} a d \\
\beta \mathrm{d} d=-q^{-1} \mathrm{~d} d \beta+\left(1-q^{-2}\right) \mathrm{d} \beta d \\
\gamma \mathrm{d} a=-q \mathrm{~d} a \gamma \quad \gamma \mathrm{d} \beta=\mathrm{d} \beta \gamma-\left(q-q^{-1}\right) \mathrm{d} a d \\
\gamma \mathrm{d} \gamma=\mathrm{d} \gamma \gamma \quad \gamma \mathrm{d} d=-q^{-1} \mathrm{~d} d \gamma+\left(1-q^{-2}\right) \mathrm{d} \gamma d \\
d \mathrm{~d} a=\mathrm{d} a d \quad d \mathrm{~d} \beta=q^{-1} \mathrm{~d} \beta d \\
d \mathrm{~d} \gamma=q^{-1} \mathrm{~d} \gamma d \quad d \mathrm{~d} d=q^{-2} \mathrm{~d} d d .
\end{gathered}
$$

Thus we have constructed the differential algebra of the algebra $\mathcal{A}$ generated by the matrix elements of any matrix in $G L_{q}(1 \mid 1)$. It is not difficult to check that the action of $\mathrm{d}$ on (24) is consistent. 
We note that the differential algebra $\Omega=\mathcal{A} \cup \mathrm{d} \mathcal{A}$ is a (graded) Hopf algebra with the following co-structure [13]: the coproduct is given by

$$
\hat{\Delta}: \Omega \longrightarrow \Omega \otimes \Omega \quad \hat{\Delta}(\hat{T})=\hat{T} \dot{\otimes} T+(-1)^{p(T)} T \dot{\otimes} \hat{T} .
$$

Explicity,

$$
\begin{aligned}
& \hat{\Delta}(\mathrm{d} a)=\mathrm{d} a \otimes a+\mathrm{d} \beta \otimes \gamma+a \otimes \mathrm{d} a-\beta \otimes \mathrm{d} \gamma \\
& \hat{\Delta}(\mathrm{d} \beta)=\mathrm{d} \beta \otimes d+\mathrm{d} a \otimes \beta+a \otimes \mathrm{d} \beta-\beta \otimes \mathrm{d} d \\
& \hat{\Delta}(\mathrm{d} \gamma)=\mathrm{d} \gamma \otimes a+\mathrm{d} d \otimes \gamma-\gamma \otimes \mathrm{d} a+d \otimes \mathrm{d} \gamma \\
& \hat{\Delta}(\mathrm{d} d)=\mathrm{d} d \otimes d+\mathrm{d} \gamma \otimes \beta-\gamma \otimes \mathrm{d} \beta+d \otimes \mathrm{d} d .
\end{aligned}
$$

The counit is given by

$$
\hat{\varepsilon}: \Omega \longrightarrow \mathcal{C} \quad \hat{\varepsilon}(\hat{T})=0
$$

and the coinverse

$$
\hat{S}: \Omega \longrightarrow \Omega \quad \hat{S}(\hat{T})=-(-1)^{p\left(T^{-1}\right)} T^{-1} \hat{T} T^{-1} .
$$

The central element is

$$
\hat{D}=b c^{-1}-\alpha c^{-1} \delta c^{-1}
$$

where $\hat{T}=\mathrm{d} T$, i.e. $\hat{D}$ commutes with the generators of $\mathcal{A}$ and also with the generators of $\hat{\mathcal{A}}$.

It is not difficult to verify that the maps $\hat{\Delta}$ and $\hat{\varepsilon}$ are both algebra homomorphisms and $\hat{S}$ is an algebra anti-homomorphism. The three maps satisfy also the properties (6)-(8), and they preserve the relations (24) provided that the actions on the generators of $\mathcal{A}$ of $\hat{\Delta}, \hat{\varepsilon}$ and $\hat{S}$ are the same as (3)-(5).

\section{The Cartan-Maurer Forms in $\Omega$}

In analogy with the right invariant one-forms defined on a Lie group in classical differential geometry, one can construct the matrix valued one-form $W$ where

$$
W=\mathrm{d} T T^{-1} .
$$

If we set

$$
T^{-1}=\left(\begin{array}{cc}
A & B \\
C & D
\end{array}\right)
$$

as the superinverse of $T \in G L_{q}(1 \mid 1)$, we write the matrix elements (one-forms) of $W$

$$
\begin{array}{ll}
w_{1}=\mathrm{d} a A+\mathrm{d} \beta C & u=\mathrm{d} a B+\mathrm{d} \beta D \\
w_{2}=\mathrm{d} d D+\mathrm{d} \gamma B & v=\mathrm{d} \gamma A+\mathrm{d} d C .
\end{array}
$$


Firstly, we obtain the commutation relations between the matrix elements of $T$ and $T^{-1}$ as follows:

$$
\begin{gathered}
a A=q^{2} A a+1-q^{2} \quad d A=A d \\
a D=D a \quad d D=q^{2} D d+1-q^{2} \\
a B=q B a \quad d B=q B d \\
a C=q C a \quad d C=q C d \\
\beta A=q A \beta \quad \gamma A=q A \gamma \\
\beta D=q D \beta \quad \gamma D=q D \gamma \\
\beta B=B \beta \quad \gamma B=-q^{2} B \gamma \\
\beta C=-q^{2} C \beta \quad \gamma C=C \gamma .
\end{gathered}
$$

Using these relations, we now find the commutation relations of the matrix entries of $T$ with those of $W$ :

$$
\begin{gathered}
a w_{1}=q^{2} w_{1} a \quad d w_{2}=q^{-2} w_{2} d+\left(q^{-2}-1\right) v \beta \\
a w_{2}=w_{2} a+\left(q^{-2}-1\right) u \gamma+\left(q-q^{-1}\right)^{2} w_{1} a \quad d w_{1}=w_{1} d \\
a u=q u a \quad d u=q^{-1} u d-\left(q-q^{-1}\right) w_{1} \beta \\
a v=q v a+\left(q-q^{-1}\right) w_{1} \gamma \quad d v=q^{-1} v d \\
\beta w_{1}=-q^{2} w_{1} \beta \quad \gamma w_{2}=-q^{-2} w_{2} \gamma+\left(1-q^{-2}\right) v a \\
\beta w_{2}=-w_{2} \beta+\left(1-q^{-2}\right) u d-\left(q-q^{-1}\right)^{2} w_{1} \beta \quad \gamma w_{1}=-w_{1} \gamma \\
\beta u=q u \beta \quad \gamma u=q^{-1} u \gamma-\left(q-q^{-1}\right) w_{1} a \\
\beta v=q v \beta+\left(q-q^{-1}\right) w_{1} d \quad \gamma v=q^{-1} v \gamma .
\end{gathered}
$$

These relations are different from those of [9] excepting a few of them.

To obtain commutation relations among the Cartan-Maurer one-forms we shall use the commutation relations between the matrix elements of $T^{-1}$ and the differentials of the matrix elements of $T$ which are given in the following

$$
\begin{aligned}
& A \mathrm{~d} a=q^{-2} \mathrm{~d} a A \quad A \mathrm{~d} d=\mathrm{d} d A \\
& A \mathrm{~d} \beta=q^{-1} \mathrm{~d} \beta A \quad A \mathrm{~d} \gamma=q^{-1} \mathrm{~d} \gamma A \\
& D \mathrm{~d} a=\mathrm{d} a D \quad D \mathrm{~d} d=q^{2} \mathrm{~d} d D+\left(q^{2}-1\right)(\mathrm{d} \gamma B-\mathrm{d} \beta C)-\left(q-q^{-1}\right)^{2} \mathrm{~d} a A \\
& D \mathrm{~d} \beta=q \mathrm{~d} \beta D+\left(q-q^{-1}\right) \mathrm{d} a B \quad D \mathrm{~d} \gamma=q \mathrm{~d} \gamma D+\left(q-q^{-1}\right) \mathrm{d} a C
\end{aligned}
$$




$$
\begin{gathered}
B \mathrm{~d} a=-q^{-1} \mathrm{~d} a B \quad B \mathrm{~d} \gamma=\mathrm{d} \gamma B+\left(q^{-2}-1\right) \mathrm{d} a A \\
B \mathrm{~d} \beta=\mathrm{d} \beta B \quad B \mathrm{~d} d=-q \mathrm{~d} d B-\left(q-q^{-1}\right) \mathrm{d} \beta A \\
C \mathrm{~d} a=-q^{-1} \mathrm{~d} a C \quad C \mathrm{~d} \beta=\mathrm{d} \beta C+\left(1-q^{-2}\right) \mathrm{d} a A \\
C \mathrm{~d} \gamma=\mathrm{d} \gamma C \quad C \mathrm{~d} d=-q \mathrm{~d} d C-\left(q-q^{-1}\right) \mathrm{d} \gamma A .
\end{gathered}
$$

We now obtain the commutation relations of the Cartan-Maurer forms, using (35) and (17), as follows:

$$
\begin{array}{cc}
w_{1} u=u w_{1} \quad u w_{2}=q^{2} w_{2} u+\left(1-q^{2}\right) w_{1} u \\
w_{1} v=v w_{1} \quad w_{2} v=q^{2} v w_{2}+\left(1-q^{2}\right) v w_{1} \\
w_{1}^{2}=0 \quad w_{2}^{2}=\left(1-q^{2}\right) v u \\
u v=q^{2} v u \quad w_{1} w_{2}+w_{2} w_{1}=\left(1-q^{2}\right) v u .
\end{array}
$$

Again, one can check that the action of $\mathbf{d}$ on (34) and also on (36) is consistent.

Note that although the Cartan-Maurer one-forms of Ref. 9 satisfy qindependent commutation relations, i.e. they are identical to the classical ones, in the present work they satisfy the $q$-dependent relations.

\section{Quantum Superalgebra}

In this section we shall obtain the deformed superalgebra of the Lie algebra generators. Firstly, we write, from (30), the Cartan-Maurer forms as

$$
\begin{array}{ll}
\mathrm{d} a=w_{1} a+u \gamma & \mathrm{d} \beta=w_{1} \beta+u d \\
\mathrm{~d} d=w_{2} d+v \beta & \mathrm{d} \gamma=w_{2} \gamma+v a .
\end{array}
$$

We can write $\mathrm{d} W$ in the form

$$
\mathrm{d} W=\sigma_{3} W \sigma_{3} W \quad \sigma_{3}=\left(\begin{array}{cc}
1 & 0 \\
0 & -1
\end{array}\right)
$$

In terms of the two-forms, these become

$$
\begin{array}{ll}
\mathrm{d} w_{1}=w_{1}^{2}-u v & \mathrm{~d} u=w_{1} u-u w_{2} \\
\mathrm{~d} w_{2}=w_{2}^{2}-v u & \mathrm{~d} v=w_{2} v-v w_{1} .
\end{array}
$$

We can now write down the Cartan-Maurer equations in our case

$$
\left(\begin{array}{cc}
\mathrm{d} w_{1} & \mathrm{~d} u \\
\mathrm{~d} v & \mathrm{~d} w_{2}
\end{array}\right)=\left(\begin{array}{cc}
-u v & q^{2}\left(w_{1}-w_{2}\right) u \\
-\left(w_{1}-w_{2}\right) v & -u v
\end{array}\right) .
$$


The commutation relations of the Cartan-Maurer forms allow us to construct the algebra of the generators. To obtain the quantum superalgebra we write down the exterior differential in the form

$$
\mathrm{d}=w_{1} T_{1}+w_{2} T_{2}+u \nabla_{+}+v \nabla_{-} .
$$

Considering an arbitrary function $f$ of the group parameters and using the nilpotency of the exterior differential $d$ one has

$$
\left(\mathrm{d} w_{i}\right) T_{i} f+\left(\mathrm{d} u_{i}\right) \nabla_{i} f=w_{i} \mathbf{d} T_{i} f-u_{i} \mathrm{~d} \nabla_{i}
$$

where

$$
w_{i} \in\left\{w_{1}, w_{2}\right\} \quad u_{i} \in\{u, v\} \quad \nabla_{i} \in\left\{\nabla_{+}, \nabla_{-}\right\} .
$$

With the Cartan-Maurer equations we find the following commutation relations for the quantum superalgebra:

$$
\begin{gathered}
{\left[T_{1}, \nabla_{+}\right]=-q^{2} \nabla_{+}+\left(q^{2}-1\right) T_{2} \nabla_{+}} \\
{\left[T_{2}, \nabla_{+}\right]=q^{2} \nabla_{+}+\left(1-q^{2}\right) T_{2} \nabla_{+}} \\
{\left[T_{1}, \nabla_{-}\right]=q^{2} \nabla_{-}+\left(1-q^{2}\right) \nabla_{-} T_{2}} \\
{\left[T_{2}, \nabla_{-}\right]=-q^{2} \nabla_{-}+\left(q^{2}-1\right) \nabla_{-} T_{2}} \\
{\left[T_{1}, T_{2}\right]=0 \quad \nabla_{ \pm}^{2}=0} \\
\nabla_{-} \nabla_{+}+q^{-2} \nabla_{+} \nabla_{-}=T_{1}+T_{2}+\left(q^{-2}-1\right)\left(T_{2}^{2}+T_{1} T_{2}\right)
\end{gathered}
$$

or with new generators $X=T_{1}+T_{2}$ and $Y=T_{1}-T_{2}$,

$$
\begin{gathered}
{\left[X, \nabla_{ \pm}\right]=0 \quad[X, Y]=0 \quad \nabla_{ \pm}^{2}=0} \\
{\left[Y, \nabla_{+}\right]=-2 q^{2} \nabla_{+}+\left(q^{2}-1\right)(X-Y) \nabla_{+}} \\
{\left[Y, \nabla_{-}\right]=2 q^{2} \nabla_{-}+\left(1-q^{2}\right) \nabla_{-}(X-Y)} \\
\nabla_{+} \nabla_{-}+q^{2} \nabla_{-} \nabla_{+}=q^{2} X+\left(1-q^{2}\right)\left(X^{2}-X Y\right) .
\end{gathered}
$$

We also note that the commutation relations (43) of the superalgebra generators should be consistent with monomials of the group parameters. To proceed, we must calculate the actions of the Leibniz rule by comparing the elements which lie together with each other from the Cartan-Maurer froms:

$$
\begin{gathered}
T_{1} a=a+q^{2} a T_{1}+\left(q-q^{-1}\right)^{2} a T_{2}+\left(q-q^{-1}\right) \gamma \nabla_{-} \\
T_{1} \beta=\beta+q^{2} \beta T_{1}-\left(q-q^{-1}\right)^{2} \beta T_{2}-\left(q-q^{-1}\right) d \nabla_{-} \\
T_{1} \gamma=\gamma T_{1}-\left(q-q^{-1}\right) a \nabla_{+} \quad T_{1} d=d T_{1}-\left(q-q^{-1}\right) \beta \nabla_{+}
\end{gathered}
$$




$$
\begin{gathered}
T_{2} a=a T_{2} \quad T_{2} d=d+q^{-2} d T_{2} \\
T_{2} \beta=\beta T_{2} \quad T_{2} \gamma=\gamma+q^{-2} \gamma T_{2} \\
\nabla_{+} a=\gamma+q a \nabla_{+}+\left(q^{-2}-1\right) \gamma T_{2} \quad \nabla_{+} d=q^{-1} d \nabla_{+} \\
\nabla_{+} \beta=d-q \beta \nabla_{+}+\left(q^{-2}-1\right) d T_{2} \quad \nabla_{+} \gamma=-q^{-1} \gamma \nabla_{+} \\
\nabla_{-} a=q a \nabla_{-} \quad \nabla_{-} d=\beta+q^{-1} d \nabla_{-}+\left(q^{-2}-1\right) \beta T_{2} \\
\nabla_{-} \beta=-q \beta \nabla_{-} \quad \nabla_{-} \gamma=a-q^{-1} \gamma \nabla_{-}+\left(q^{-2}-1\right) a T_{2} .
\end{gathered}
$$

Notice that these commutation relations must be consistent. In fact, it is easy to see that the nilpotency of $\nabla_{ \pm}^{2}=0$ is consistent with

$$
\nabla_{ \pm}^{2} a=q^{2} a \nabla_{ \pm}^{2}
$$

Also, the commutation relations of $T_{1}, T_{2}$ with $\nabla_{-}$are consistent with

$$
\begin{gathered}
\left(\left[T_{1}, \nabla_{-}\right]-q^{2} \nabla_{-}+\left(q^{2}-1\right) \nabla_{-} T_{2}\right) a=q a\left(\left[T_{1}, \nabla_{-}\right]-q^{2} \nabla_{-}+\left(q^{2}-1\right) \nabla_{-} T_{2}\right) \\
\left(T_{2} \nabla_{-}-q^{2} \nabla_{-} T_{2}+q^{2} \nabla_{-}\right) a=q a\left(T_{2} \nabla_{-}-q^{2} \nabla_{-} T_{2}+q^{2} \nabla_{-}\right) .
\end{gathered}
$$

Furthermore,

$$
\left(T_{1} T_{2}-T_{2} T_{1}\right) a=\left(q^{-3}-q^{-1}\right) \gamma\left(T_{2} \nabla_{-}-q^{2} \nabla_{-} T_{2}+q^{2} \nabla_{-}\right)
$$

and

$$
\begin{aligned}
\left(\nabla_{-} \nabla_{+}+\right. & \left.q^{-2} \nabla_{+} \nabla_{-}-T_{1}-T_{2}-\left(q^{-2}-1\right)\left(T_{2}^{2}+T_{1} T_{2}\right)\right) a \\
& =q^{2} a\left(\nabla_{-} \nabla_{+}+q^{-2} \nabla_{+} \nabla_{-}-T_{1}-T_{2}-\left(q^{-2}-1\right)\left(T_{2}^{2}+T_{1} T_{2}\right)\right) .
\end{aligned}
$$

Similarly, one can find the other relations.

\section{R-matrix approach}

In this section we wish to obtain the relations (17), (24), (34) and (36) with the help of a matrix $\hat{R}$ that acts on the square tensor space of the supergroup. Of course, the matrix $\hat{R}$ is a solution of the quantum (graded) braided group equation.

We first consider the quantum superplane and its dual [5]. The quantum superplane $A_{q}$ is generated by coordinates $x$ and $\theta$, and the commutation rules

$$
x \theta=q \theta x \quad \theta^{2}=0 .
$$


The quantum (dual) superplane $A_{q}^{*}$ is generated by coordinates $\varphi$ and $y$, and the commutation rules

$$
\varphi^{2}=0 \quad \varphi y=q^{-1} y \varphi
$$

We demand that relations (46), (47) are preserved under the action of $T$, as a linear transformation, on the quantum superplane and its dual:

$$
T: A_{q} \longrightarrow A_{q} \quad T: A_{q}^{*} \longrightarrow A_{q}^{*}
$$

Let $X=(x, \theta)^{t}$ and $\hat{X}=(\varphi, y)^{t}$. Then, as a consequence of (48) the points $T X$ and $T \hat{X}$ should belong to $A_{q}$ and $A_{q}^{*}$, respectively, which give the relations (2).

Similarly, let us consider linear transformations $\hat{T}$ with the following properties

$$
\hat{T}: A_{q} \longrightarrow A_{q}^{*} \quad \hat{T}: A_{q}^{*} \longrightarrow A_{q} .
$$

Then the points $\hat{T} X$ and $\hat{T} \hat{X}$ should belong to $A_{q}^{*}$ and $A_{q}$, respectively. This case is equivalent to (17).

Note that the relations (46) can be written as follows

$$
X \otimes X=q^{-1} \hat{R} X \otimes X
$$

where

$$
\hat{R}=\left(\begin{array}{cccc}
q & 0 & 0 & 0 \\
0 & q-q^{-1} & 1 & 0 \\
0 & 1 & 0 & 0 \\
0 & 0 & 0 & -q^{-1}
\end{array}\right)
$$

We can also write mixed relations between the component of $X$ and $\hat{X}$ as follows:

$$
(-1)^{p(X)} X \otimes \hat{X}=q \hat{R} \hat{X} \otimes X
$$

where $\hat{X}=\mathrm{d} X$.

Using (48) together with (50) and (52), we now derive anew the quantum supergroup relations (2) from the equation [9]

$$
\hat{R} T_{1} T_{2}=T_{1} T_{2} \hat{R}
$$

where, in usual grading tensor notation, $T_{1}=T \otimes I$ and $T_{2}=I \otimes T$. Similarly using (52), we obtain the following equation

$$
(-1)^{p\left(T_{1}\right)} T_{1} \hat{T}_{2}=\hat{R} \hat{T}_{1} T_{2} \hat{R}
$$


which is equivalent to the relations (24). The equation

$$
\hat{T}_{1}^{\prime} \hat{T}_{2}=(-1)^{p\left(\hat{T}_{1}\right)} \hat{R} \hat{T}_{1} \hat{T}_{2} \hat{R} \quad \hat{T}_{1}^{\prime}=\mathrm{d}\left((-1)^{p\left(T_{1}\right)} T_{1}\right)
$$

gives the relations (17). Taking $\hat{T}=W T$ and using (55) one has

$$
(-1)^{p\left(T_{1}\right)} T_{1} W_{2}=\hat{R} W_{1} \hat{R} T_{1}
$$

which gives the relations (34). Finally, from (55) we find that

$$
(-1)^{p\left(W_{1}\right)} W_{1} \hat{R} W_{1} \hat{R}^{-1}+(-1)^{p\left(W_{1}\right)} \hat{R} W_{1} \hat{R} W_{1}=0 .
$$

This equation is equivalent to (36).

\section{Discussion}

The present paper may be considered as an alternative to the approach proposed earlier by Schmidke et al [9]. They have consructed a right-invariant differential calculus on the quantum supergroup $G L_{q}(1 \mid 1)$ and showed that the quantum superalgebra generators satisfy the undeformed Lie superalgebra. Their starting point is to evaluate the $q$-commutation relations of the group parameters [the matrix elements of the matrix in $G L_{q}(1 \mid 1)$ ] with the Cartan-Maurer forms, directly. They have assumed that "the right action of the group suggests that $a$ and $\beta$, and, $\gamma$ and $d$ [the matrix elements in (1)] satisfy the same relations as the forms". The starting point of the present paper, however, is to evaluate the $q$-commutation relations of the matrix elements with their differentials. Later, using these relations the $q$-commutation relations of the matrix elements with the Cartan-Maurer forms are obtained without any further assumptions. In the work of [9], the commutation relations of the Cartan-Maurer forms are obtained by using the $q$-commutation relations of the matrix elements with the Cartan-Maurer forms, i.e., to obtain the desired commutation relations they have applied the exterior differential $\delta$ on the relations of the matrix elements with the Cartan-Maurer forms. In the present work we also use the same approach. But although the CartanMaurer forms in [9] satisfy $q$-independent relations, in the present paper they satisfy $q$-dependent relations. Since in [9], the Cartan-Maurer forms satisfy undeformed [ $q$-independent $]$ relations, the quantum Lie algebra obtained is also undeformed.

The work of [9] does not allow an R-matrix approach. In our work we have derived the $q$-commutation relations between the matrix elements and their differentials without considering an R-matrix at first. However we later show that these relations can also be derived using an R-matrix. This R-matrix 
turns out to be the same as used by Wess and Zumino [7] for the commutation relations between coordinates and their differentials for the $G L_{q}(2)$ invariant calculus on the $q$-plane.

\section{Acknowledgments}

This work was supported in part by T. B. T. A. K. the Turkish Scientific and Technical Research Council. We would like to express our deep gratitude to the referees for critical comments and suggestions on the maniscript.

\section{References}

[1] Drinfeld V G 1986 Quantum groups in Proc Int Cong Math Berkeley 798 Reshetikhin N Y, Takhtajan L A and Faddeev L D 1990 Leningrad Math J 1193

[2] Faddeev L D 1990 From integrable models to quantum groups Schladming Lectures

Alvarez-Gaume L, Gomes C and Sierra G 1989 Nucl Phys B 319155

Curtright T, Fairlie D and Zachos C 1990 eds Quantum groups Proc Argonne Workshop (World Scientific)

Fairlie D B and Zachos C K 1991 Phys Lett B 25643

[3] Banks T 1997 Matrix Theory Preprint (RU-97-76)

Bigatti D and Susskind L 1997 Review of Matrix Theory (SU-ITP-97-51)

[4] Manin Yu I 1989 Commun Math Phys 123163

[5] Woronowicz S L 1989 Commun Math Phys 122125

[6] Connes A 1991 Noncommutative Geometry (Academic Press)

[7] Wess J and Zumino B 1990 Nucl Phys B 18302

[8] Schirrmacher A, Wess J and Zumino B 1991 Z Phys C 49317

Schupp P, Watts P and Zumino B 1992 Lett Math Phys 25139

Isaev A P 1994 J Math Phys 356784 Dubois-Violette M, Kerner R and Madore J 1990 J Math Phys 31316

[9] Schmidke W, Vokos S and Zumino B 1990 Z Phys C 48249 
[10] Corrigan E, Fairlie D, Fletcher P and Sasaki R 1990 J Math Phys 31776

[11] Schwenk S, Schmidke W and Vokos S 1990 Z Phys C 46643

[12] Celik S and Celik S A 1995 Balkan Phys Lett 3188

[13] Celik S 1997 "Hopf algebra structure of $G r_{q}(1 \mid 1)$ related to $G L_{q}(1 \mid 1)$ " Preprint MSUMB 97-09 